\title{
Effect of Adaptation on Object Representation Accuracy in Macaque Inferior Temporal Cortex
}

\author{
Dzmitry A. Kaliukhovich ${ }^{1}$, Wouter De Baene ${ }^{2}$, and Rufin Vogels ${ }^{1}$
}

\begin{abstract}
Stimulus repetition produces a decrease of the response in many cortical areas and different modalities. This adaptation is highly prominent in macaque inferior temporal (IT) neurons. Here we ask how these repetition-induced changes in IT responses affect the accuracy by which IT neurons encode objects. This question bears on the functional consequences of adaptation, which are still unclear. We recorded the responses of single IT neurons to sequences of familiar shapes, each shown for $300 \mathrm{msec}$ with an ISI of the same duration. The difference in shape between the two successively presented stimuli,that is, adapter and test, varied parametrically. The discriminability of the test stimuli was reduced for repeated compared with nonrepeated stimuli. In some conditions for which adapter and test shapes differed, the cross-adaptation resulted
\end{abstract}

\section{INTRODUCTION}

The responses of many neurons in macaque inferior temporal (IT) cortex, a visual cortical area coding for object properties, decrease with stimulus repetition (e.g., Kaliukhovich \& Vogels, 2011, 2012; De Baene \& Vogels, 2010; Liu, Murray, \& Jagadeesh, 2009; McMahon \& Olson, 2007; Sawamura, Orban, \& Vogels, 2006; Miller \& Desimone, 1994; Miller, Gochin, \& Gross, 1991; Miller, Li, \& Desimone, 1991; Baylis \& Rolls, 1987; Gross, Bender, \& Rocha-Miranda, 1969; Gross, Schiller, Wells, \& Gerstein, 1967). This "repetition suppression" (Desimone, 1996) or "adaptation" effect (Ringo, 1996) has aroused recent interest because of the widespread use of adaptation paradigms in fMRI studies (Grill-Spector, Henson, \& Martin, 2006). Previous studies suggested that adaptation of spiking activity of single IT neurons results from suppression of the input and/or synaptic depression (De Baene \& Vogels, 2010; Liu et al., 2009; Sawamura et al., 2006), at least in paradigms that use short adapter durations and ISIs, therefore assessing short-term adaptation effects.

Here we ask how short-term stimulus repetition affects the accuracy of object coding in IT cortex. This is an important question, because it addresses the functional consequences of adaptation in IT, which, as in other areas (Kohn, 2007), remain unclear. Studies in earlier visual

${ }^{1}$ KU Leuven, ${ }^{2}$ Universiteit Gent in an enhanced discriminability. These single cell results were confirmed in a second experiment in which we recorded multiunit spiking activity using a laminar microelectrode in macaque IT. Two familiar stimuli were presented successively for $500 \mathrm{msec}$ each and separated with an ISI of the same duration. Trials consisted either of a repetition of the same stimulus or of their alternation. Small neuronal populations showed decreased classification accuracy for repeated compared with nonrepeated test stimuli, but classification was enhanced for the test compared with adapter stimuli when the test stimulus differed from recently seen stimuli. These findings suggest that short-term, stimulus-specific adaptation in IT supports efficient coding of stimuli that differ from recently seen ones while impairing the coding of repeated stimuli. areas have reported that adaptation improves stimulus discriminability (Hansen \& Dragoi, 2011; Wang, Iliescu, Ma, Josic, \& Dragoi, 2011; Gutnisky \& Dragoi, 2008; Müller, Metha, Krauskopf, \& Lennie, 1999), in agreement with Barlow (1961). Given that repetition suppression appears to be stronger in IT than in earlier regions (Orban \& Vogels, 1998), it is unclear whether stimulus coding accuracy in IT increases for adapted stimuli. We examined this issue by measuring the stimulus discriminability of IT neurons following adaptation by reanalyzing data of two experiments: one in which responses of single IT neurons were measured with parameterized shapes (De Baene \& Vogels, 2010) and a second one in which multiunit activity (MUA) was measured simultaneously with up to 16 electrodes using a laminar probe (Kaliukhovich \& Vogels, 2012).

\section{METHODS}

\section{Subjects}

Two rhesus macaques (Macaca mulatta) participated in each experiment with one animal being a subject in both. Animal care and experimental procedures met the national and European guidelines and were approved by the ethical committee of the KU Leuven Medical School. Details about implants and surgery can be found in Kaliukhovich and Vogels (2012) and De Baene and Vogels (2010). The single cells were recorded in both 
the lateral convexity of IT and lower bank of the superior temporal sulcus (STS) with $\mathrm{A} / \mathrm{P}$ levels ranging between 11 and $22 \mathrm{~mm}$ anterior to the auditory meatus. The MUA recordings with a laminar probe were made from the lower bank of the STS with A/P levels ranging between 15 and $18 \mathrm{~mm}$ anterior.

\section{Recordings}

Single units were recorded with tungsten microelectrodes and discriminated on-line (see De Baene \& Vogels, 2010, for details). MUA was recorded using a 16-channel Plextrode U-Probe (Plexon, Inc.) with an intercontact (electrode site) spacing of $100 \mu \mathrm{m}$ and electrode sites being linearly arranged on a single shaft. The MUA was thresholded on-line, and the thresholded spike waveforms were saved at $40 \mathrm{kHz}$ (see Kaliukhovich \& Vogels, 2012, for details). The U-Probe was positioned in the STS so that visually driven MUA was present on most if not all channels. After positioning the U-Probe, we waited for approximately $2 \mathrm{hr}$ before performing the recordings to ensure good recording stability. In both experiments, the grounded guide tube served as the reference and recordings were made with a Plexon data acquisition system.

Eye positions were measured on-line with an infraredbased eye tracking system (ISCAN, Inc.; $120 \mathrm{~Hz}$ sampling rate). The eye movement signal was saved with a sampling frequency of $1 \mathrm{kHz}$. Eye positions, stimulus, and behavioral events were stored for off-line analysis on a computer.

\section{Stimuli and Tests}

\section{Single Unit Recordings Experiment}

The stimuli and tests were described in detail by De Baene and Vogels (2010) and, thus, will only be briefly summarized here. We employed four sets of shaded 3-D shapes. Each set consisted of six shapes that were created by morphing one shape toward another (Figure 2A of De Baene \& Vogels, 2010). One of the shape sets is shown in Figure 1A. The shape heights ranged from $5.4^{\circ}$ to $7.2^{\circ}$, and their area and mean luminance were equated. The shapes were presented foveally on a gray background.

In a preliminary test, the shape selectivity of an isolated IT neuron was assessed using all 24 stimuli ( 4 sets $\times$ 6 shapes). On the basis of inspection of the on-line peristimulus time histograms (PSTH) of the responses, a shape set for which the neuron showed shape selectivity was selected. Then we ran the adaptation test (Figure 1B) in which each of the six shapes (adapters) could be followed by each of the six shapes (test stimuli) of the selected shape set. Thus, we presented all 36 possible adaptation sequence conditions (sequences A-A, A-B, A-C, ..., F-E, F-F). The stimulus duration and ISI were $300 \mathrm{msec}$, and stimuli were presented during monkey's stable fixation of a $0.18^{\circ}$ red target (fixation window $\leq 2^{\circ}$ ). Trials in which the monkey aborted fixation were not analyzed further.

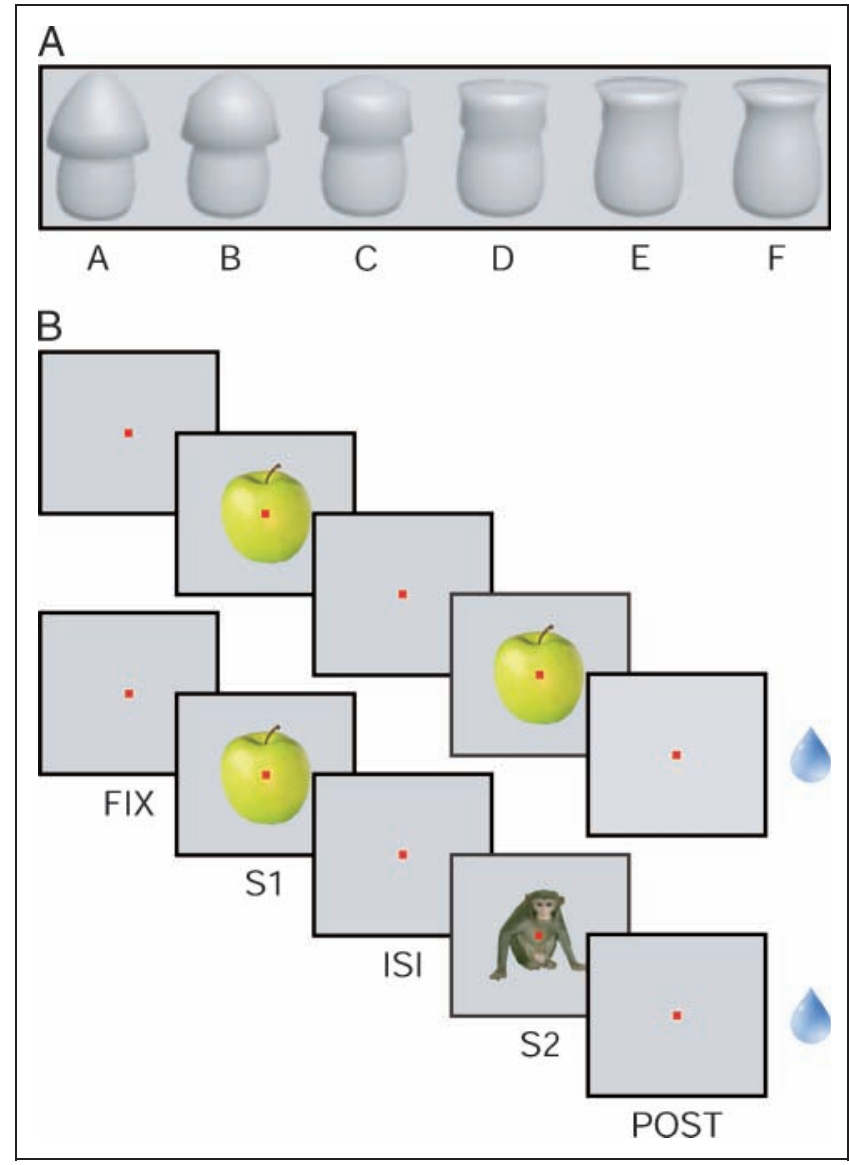

Figure 1. Stimuli and adaptation paradigm. (A) Illustration of one set of shapes employed in the single cell study (Experiment 1). The letters serve to identify each of the shapes. (B) Schematic of adaptation test with two of the images employed in the simultaneous MUA recordings study (Experiment 2). Top and bottom illustrate a repetition and alternation trial, respectively. In both experiments, the animals were required to fixate a small fixation target (shown here not to scale) presented at the center of a monitor for $500 \mathrm{msec}$ before stimulus onset (FIX). The adapter (S1) and test (S2) stimuli were shown for 300 and $500 \mathrm{msec}$ in Experiments 1 and 2, respectively, and separated by an ISI of the same duration as the stimulus. Monkeys were required to fixate during the entire trial, including the poststimuli (POST) interval, lasting 300 and 475 msec in Experiments 1 and 2, respectively. Successful fixation throughout a trial was rewarded by a drop of apple juice.

The sequences were shown in pseudorandom order so that a stimulus presented in adaptation trial $n$ did not reoccur in adaptation trial $n+1$. To unadapt the neuron, two trials in which two randomly chosen scrambled versions of the 3-D shapes were presented followed each adaptation trial. The mean number of unaborted trials per condition was 8.11 across neurons.

\section{Simultaneous MUA Recordings Experiment}

The stimuli and tests were described in detail by Kaliukhovich and Vogels (2012) and will only be briefly summarized here. The stimuli consisted of 52 color images (maximum size approximately $5^{\circ}$ ) of animate and inanimate objects including two fractals that were presented 
foveally on a gray background. On the basis of the on-line MUA PSTHs of the responses to all 52 stimuli in a preliminary test, in each penetration we selected two images that elicited a response in several (if not all) of the channels. Across the 32 penetrations, the majority of the selected stimuli included inanimate objects (63\%), whereas a fractal was selected only twice (3\%). Then, the selected two stimuli, adapter and test, were presented for $500 \mathrm{msec}$ each, with an ISI of $500 \mathrm{msec}$ during stable fixation of a $0.17^{\circ}$ red target (fixation window $\leq 2.6^{\circ}$ ). The stimuli within a trial were either the same (AA or BB; repetition trials) or different ( $\mathrm{AB}$ or $\mathrm{BA}$; alternation trials; Figure 1B) images. The order of the trial sequences was pseudorandomized with the constraint that the adapter image of a trial always differed from the last presented image of the preceding unaborted or aborted trial. Aborted trials were not analyzed further. The mean number of unaborted trials per condition was 123.5 across penetrations.

\section{Data Analysis}

Responses to Stimuli

A single neuron or MUA site was considered to be responsive to a particular stimulus if the average firing rate to that stimulus as adapter or test was significantly greater than the average baseline firing rate (two-sided Wilcoxon matched pairs test: $p<.05)$. The baseline firing rate was measured within a 300- (single unit) or 200-msec (MUA) analysis window that started 300 (single unit) or $140 \mathrm{msec}$ (MUA) before the adapter stimulus onset. Firing rates for the adapter and test stimuli were computed within 300 (single unit) or 500 (MUA) msec windows starting 50 (single unit) or $60 \mathrm{msec}$ (MUA) after stimulus onset.

All analyses were performed on responses defined as raw spike counts. For the single units, spike counts were measured using a window of $300 \mathrm{msec}$ (equal to the stimulus duration) that started $50 \mathrm{msec}$ after stimulus onset, which is the same analysis window as in De Baene and Vogels (2010). Since a previous analysis of the MUA recordings (Kaliukhovich \& Vogels, 2012) showed that the adaptation was stronger in the early than in the late phase of the response to a stimulus, we employed in most analyses of the MUA a window of 60- to 310-msec poststimulus onset (stimulus duration was $500 \mathrm{msec}$ ).

\section{Stimulus Discriminability Metric}

For each responsive single neuron and MUA site, we computed a discriminability index $d^{\prime}$ defined as

$$
d^{\prime}=\operatorname{abs}(M(\mathrm{P})-M(\mathrm{~L})) / \sqrt{ }\left(\sigma^{2}(\mathrm{P})+\sigma^{2}(\mathrm{~L})\right) / 2
$$

with $M(X)$ and $\sigma(X)$ being the mean and standard deviation of the response to stimulus $X$, respectively. For each of the single units, we first determined their preferred shape (of the six shapes constituting a set) by averaging the responses across the six conditions that had the same adapter shape and selecting the shape that produced the maximum averaged response. Then we defined $\mathrm{P}$ as the preferred shape and $\mathrm{L}$ as the shape that differed either by 1,2 , or 3 steps (values) along the morph dimension from the preferred shape. Maximally, a distance of three values was chosen because this is the maximum possible distance for neurons for which the preferred shape was shape $\mathrm{C}$ or D of Figure $1 \mathrm{~A}$. The shapes with a distance of 1 or 2 with respect to the preferred shape were inbetween the preferred shape and the shape with a distance of 3 . In the main analysis, $d^{\prime}$ scores were computed for three pairs of shapes of which one member was the preferred shape and the other member was the shape that differed by, respectively, 1, 2, and 3 values from the preferred shape. In the MUA recordings, only two stimuli were presented in each penetration, and thus, $d^{\prime}$ was computed for this pair of stimuli by necessity. The $d^{\prime}$ values were computed separately for different conditions, which will be explained in the Results.

\section{Classification of Simultaneously Recorded MUA}

We classified the two stimuli, A and B, using the simultaneously recorded MUA population (maximum 16 MUA sites) for each penetration separately with a correlationbased classifier (Meyers, Freedman, Kreiman, Miller, \& Poggio, 2008). Results were qualitatively similar to those obtained when the stimuli were classified with Support Vector Machines (for discussion of the advantages of the correlation-based classifier, see Meyers et al., 2008). Classification was performed with sliding windows of varying length (12.5-100 msec) with a step size of $10 \mathrm{msec}$ or with the same $250 \mathrm{msec}$ response window as used to compute $d^{\prime}$. To compare classification accuracy across conditions (see Results), we equated for these conditions the number of trials per stimulus that were used to train and test the classifier. Thus, for each penetration, the number of trials per stimulus $N_{\text {tot }}$ that entered the classifier was equal to the smallest even number of trials per stimulus of the conditions of that penetration. The mean $N_{\text {tot }}$ was 112 trials and ranged from 84 to 122 . Half of the $N_{\text {tot }}$ trials were used for training, whereas the remaining half of the trials was used for testing the classifier. All the reported classification accuracies are based on the classifications obtained during testing (cross-validated classification scores). For each condition and penetration, we trained and tested 1000 classifiers by randomly drawing for each classifier the training and test trials from the pool of all available trials. The reported classification scores of a population of neurons of a penetration are the averages of these 1000 classification scores.

The correlation coefficient-based classifier correlates the response vector (i.e., the simultaneously recorded responses at the different sites of a penetration) of a test trial with each of the mean response vectors of the two stimuli $\mathrm{A}$ and $\mathrm{B}$ (i.e., the mean responses-averaged 
across training trials-of the sites). The test trial is classified as the stimulus for which this correlation is the highest. The training and test data for each site were standardized by subtracting the mean (averaged across the stimuli for the training trials) from each response and dividing this difference by the standard deviation of the responses of the training data. As a control, the stimulus labels (A or B) were randomly permuted across the trials. This permutation of the stimulus labels was performed 1000 times, and 1000 classifiers were trained. As expected, the mean percent correct classification accuracy for the shuffled data was $50 \%$.

In addition to training and testing classifiers for a given condition (e.g., training and testing the data for the test stimuli in repetition trials), we also determined how well one can classify the stimuli in the different conditions when the training data consisted of responses to the adapter stimuli only. For this adapter-based classification, adapter presentations were employed for training, and testing was done using independent adapter presentations and the test stimulus presentations of the other conditions. The numbers of trained and test trials were kept equal to that employed for the other classifications.

\section{Eye Movement Analyses}

Kaliukhovich and Vogels (2012) and De Baene and Vogels (2010) analyzed eye movements for the adapter and test stimuli of the experiments reported on in this paper and showed that the stimulus-selective repetition suppression of the neural responses cannot be explained by eye movement differences between the adapter and test stimuli.

\section{RESULTS}

\section{Effect of Adaptation on Shape Discriminability of Single IT Neurons}

We assessed the effect of stimulus repetition on the stimulus discriminability of macaque IT neurons in two different experiments. In the first experiment (De Baene \& Vogels, 2010), single unit responses ( $n=80$ responsive neurons; 40 in each animal) were measured for adapter and test stimuli that varied systematically in shape. As reported by De Baene and Vogels (2010), the response to the test stimulus was reduced by a median value of $22 \%$ with respect to the response to the adapter. The critical question, however, is what effect this repetition suppression has on the ability of single IT neurons to signal shape identity?

For each neuron, we determined its preferred shapeamong the six tested shapes-and then computed $d^{\prime}$ scores for shape pairs that included the preferred shape $\mathrm{P}$ and another shape $\mathrm{L}$, which varied in distance $(D)$ to $\mathrm{P}$. We computed $d^{\prime}$ s for each of three distances along the morph dimension between $\mathrm{P}$ and L. For instance, if the preferred shape $\mathrm{P}$ of a particular neuron had a value of 3 on the morph dimension, then $\mathrm{L}$ could have values of $4(D=1), 5(D=2)$, and $6(D=3)$. For each shape pair, $d$ 's were computed for five different adaptation conditions. The first condition was based on the responses to the shapes presented as adapter irrespective of the second stimulus, that is, $\mathrm{P}$ versus $\mathrm{L}$ presented as the first stimulus of a sequence (trial). The other four conditions were based on responses to the shapes presented as test stimuli, that is, as the second stimulus of a sequence, sorted according to the first stimulus of a sequence. These four conditions consisted of $d^{\prime}$ s computed for $\mathrm{P}$ versus $\mathrm{L}$ as test stimuli in (1) trials when both stimuli were repeated (PP vs. LL or Test $(\mathrm{PP}, \mathrm{L} \mathbf{L})),(2)$ trials when the two stimuli alternated (Test $(\mathrm{P} \mathbf{L}, \mathbf{L} \mathbf{P}))$, (3) trials in which $\mathrm{P}$ was repeated versus $\mathrm{L}$ following $\mathrm{P}$ as adapter (Test $(\mathrm{P} \mathbf{P}, \mathrm{P} \mathbf{L}))$, and (4) trials in which $\mathrm{L}$ was repeated versus $\mathrm{P}$ following $\mathrm{L}$ as adapter (Test (LL, LP)). Because, as expected, the mean $d^{\prime}$ s for the adapter in repetition and alternation trials were not statistically different for each of the three distances (two-sided Wilcoxon matched pairs tests: $p>$.7154), we pooled the $d^{\prime}$ 's for the adapter in repetition and alternation trials for each distance for each neuron. We pooled the data of the two animals because the adaptation effects were similar in both animals (De Baene \& Vogels, 2010).

Figure 2A shows the mean $d^{\prime}$ for $\mathrm{P}$ versus $\mathrm{L}$ for three different shape distances along the morph dimension as a function of the five conditions. As expected and validating our shape distance manipulation, $d^{\prime}$ decreased with decreasing shape distance (repeated-measures ANOVA: main effect of Distance: $F(2,158)=39.746, p=.1044$. $\left.10^{-13}\right)$. The $d^{\prime}$ values were highly influenced by the adaptation condition (ANOVA: main effect of Adaptation condition: $\left.F(4,316)=17.061, p=.1112 \cdot 10^{-11}\right)$, and this was true at each distance (ANOVA: interaction Distance and Condition: $F(8,632)=1.6464, p=.1085)$. Post hoc Bonferroni tests showed a decrease in $d^{\prime}$ for the test in repetition trials (Test $(\mathrm{P} \mathbf{P}, \mathrm{L} \mathbf{L}))$ and the Test $(\mathrm{P} \mathbf{P}, \mathrm{PL})$ condition compared with the response to the adapter and the response to the test in alternation trials (Test $(\mathrm{PL}$, LP); all $p$ values < .0004). Adaptation to $\mathrm{P}($ Test $(\mathrm{PP}, \mathrm{PL}))$ reduced the $d^{\prime}$, whereas adaptation to $\mathrm{L}(\operatorname{Test}(\mathrm{L} \mathbf{L}, \mathbf{L P}))$ tended to enhance the $d^{\prime}$.

The $d^{\prime}$ values are determined by both the differences in response to $\mathrm{P}$ and $\mathrm{L}$ and the variability of the responses to $\mathrm{P}$ and $\mathrm{L}$. Table 1 shows the mean responses and mean standard deviations of the responses (averaged across the 80 neurons) and mean Fano factors (response variance/ mean response) for the $\mathrm{P}$ and $\mathrm{L}$ stimuli presented as adapter and for the $\mathrm{P}$ and $\mathrm{L}$ stimuli presented as a test and this as a function of the preceding adapter. The shown values are for a shape distance of 2 . The row "SUA: Mean (Adapter-Test)" in Table 1 indicates the response difference between a shape presented as a test and the same shape presented as adapter, with positive values indicating a reduction of the response to the test compared with the 


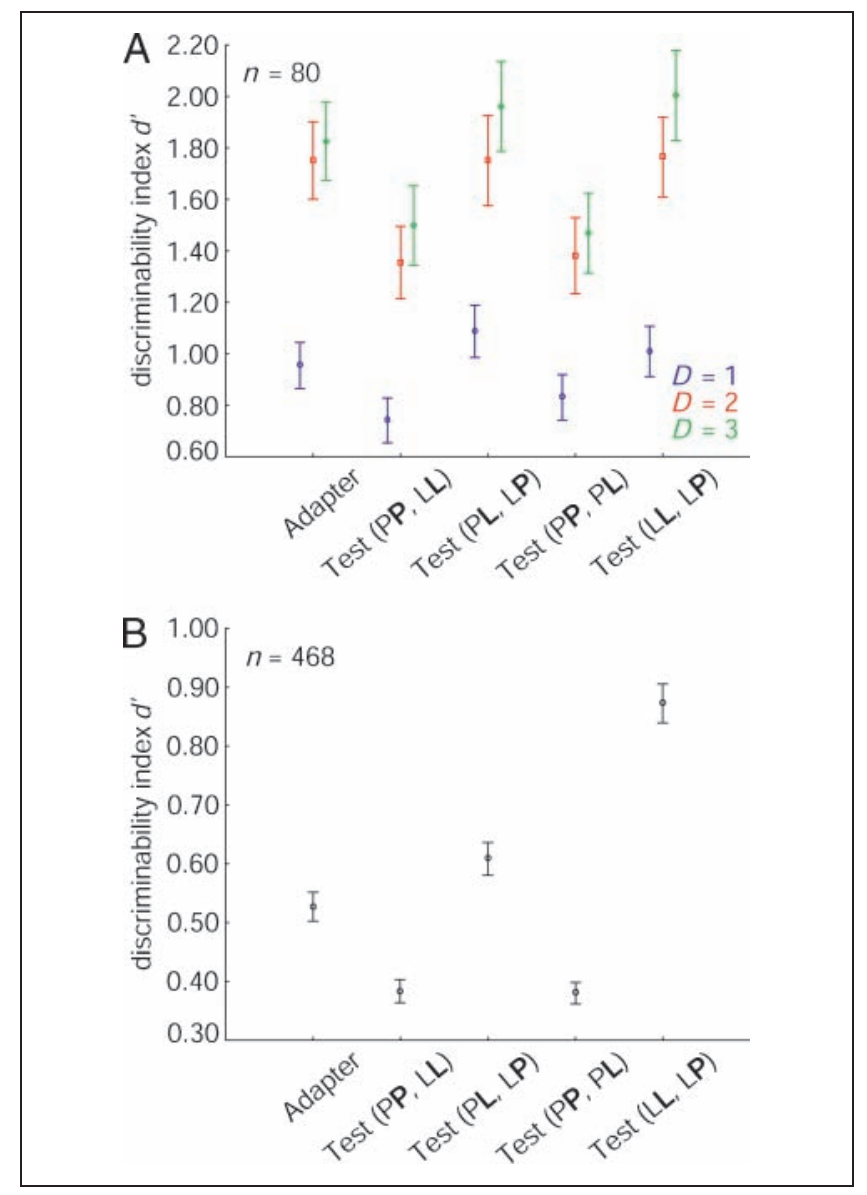

Figure 2. Mean discriminability index $d^{\prime}$ for adapter and test stimuli across different adaptation conditions. (A) Mean $d^{\prime}$ of single neurons $(n=80)$ measured at three different distances (D) between the preferred (P) and less preferred (L) shapes for five different conditions: shapes presented as adapter, shapes presented as test in repetition trials (Test $(\mathbf{P P}, \mathrm{L} \mathbf{L}))$, shapes presented as test in alternation trials (Test(PL, LP)), and shapes presented as test of which one was following an identical adapter and the other shape was following the different adapter (Test(PP, PL) and Test(LL, LP)). (B) Mean $d^{\prime}$ of MUA activity ( $n=468$ responsive sites) for the same five conditions as in A. Each MUA site was stimulated by two stimuli $\mathrm{P}$ and $\mathrm{L}$ that were defined as the stimuli eliciting the largest and smallest response, respectively, when presented as adapter. In both A and B, bars indicate standard errors of the mean and bold letters correspond to the stimulus pairs for which $d^{\prime}$ was computed.

adapter. The response reduction was larger for the P compared with L stimuli presented as tests in repetition trials (Test(P) vs. Test(LL)). This naturally follows from the observation that the degree of repetition suppression in IT is relatively constant for different stimuli when expressed as a ratio between the responses to the test and adapter stimuli (De Baene \& Vogels, 2010; Liu et al., 2009; Sawamura et al., 2006). However, it also implies that the difference in mean response between $\mathrm{L}$ and $\mathrm{P}$ as test stimuli in repetition trials will be smaller than the differences between the same stimuli presented as adapter, which can explain the lower $d^{\prime}$ for test compared with adapter stimuli in repetition trials (i.e., Figure $2 \mathrm{~A}, d^{\prime}$ in the Test (PP, LL) condition is lower than that of the adapter).
Table 2 shows the mean differences in response between $\mathrm{L}$ and $\mathrm{P}$ for the five adaptation conditions. The response differences were computed signed (first row; based on first row of Table 1) or as absolute differences (second row; as when computing $d^{\prime}$ ). The lower signed compared with absolute response differences are due to a minority of neurons that changed their tuning for the test compared with the adapter shape in the cross-adaptation conditions. The absolute response differences covary with the $d^{\prime}$ values (third row of Table 2), indicating that the adaptation effects are largely determined by changes of the mean response and minimally by the slight changes in response variance (Table 1 ). The reduced $d^{\prime}$ in the Test $(\mathbf{P} \mathbf{P}, \mathrm{P} \mathbf{L})$ compared with adapter condition results from the stronger reduction of the response when repeating $\mathrm{P}$ than when $\mathrm{L}$ is following $\mathrm{P}$. This stronger reduction in the former condition is not merely because L produces a smaller response than $\mathrm{P}$, because the response to $\mathrm{L}$ following L is still smaller than that to L following P (Table 1). It does, however, reflect the larger suppression of the responses in repetition compared with alternation trials (De Baene \& Vogels, 2010; Liu et al., 2009). Thus, the observed $d^{\prime}$ s can be explained by the adaptation-induced reductions of the mean responses in the different stimulus conditions.

The Müller et al. (1999) V1 study observed an enhanced discriminability in the Test(LL, LP) condition compared with the adapter. A similar, but nonsignificant, trend was present in the IT data (post hoc $t$ test: $p=.22$ ), most pronounced for the largest shape distance (Figure 2A). However, our analysis shows that a possible enhanced discriminability for this condition cannot be generalized to other adaptation conditions, since the Test $(\mathrm{P} \mathbf{P}, \mathrm{P} \mathbf{L})$ condition showed a strong reduction in discriminability. Dragoi, Sharma, Miller, and Sur (2002) observed that a 400-msec adaptation to grating enhanced the $d^{\prime}$ around the preferred orientation of V1 neurons for large differences $\left(>45^{\circ}\right)$ between the adapter and the preferred orientation. To determine whether IT neurons show a similar effect, we selected those neurons for which the preferred shape was either one of the extremes of the shape dimension or differed by one value from those extremes (i.e., shapes A, B, E, and F in Figure 1A), and which also showed a response difference between the preferred shape and the other extreme shape that was at least a factor of 1.5. The $d^{\prime}$ s were computed for the preferred shape and the shape differing by two values (i.e., distance $=$ 2 ). For each neuron, the $d^{\prime}$ s were computed for the same shape pairs presented as adapter and as test following each of five adapters. The five adapters differed in their value along the morph dimension, ranging from identical to the preferred shape (adapter value 1 in Figure 3 ) to a shape differing by four values from the preferred shape (adapter value 5 in Figure 3). The $d^{\prime}$ s for the adapter and test conditions were computed for independent trials. Figure 3A shows the mean $d^{\prime}$ ( $n=26$ neurons) for the five test conditions as a function of the adapter value 
Table 1. Mean Response, Response Averaged across Neurons; Mean Standard Deviation, Between-trial Standard Deviation Averaged across Neurons; Mean Fano Factor, Fano Factor (Response Variance/Mean) Averaged across Neurons; Mean (Adapter - Test), Average across Neurons of the Difference between Response to the Same Stimulus Presented as Adapter and as Test

\begin{tabular}{|c|c|c|c|c|c|c|}
\hline & Adapter $\boldsymbol{P}$ & $\operatorname{Test}(L \boldsymbol{P})$ & Test $($ PP $)$ & Adapter $\boldsymbol{L}$ & Test $(P \boldsymbol{L})$ & Test $(L \boldsymbol{L})$ \\
\hline SUA: Mean response, spikes/sec & 49.10 & 39.87 & 34.72 & 33.71 & 27.52 & 25.34 \\
\hline SUA: Mean standard deviation, spikes/sec & 11.10 & 11.27 & 10.73 & 9.63 & 8.87 & 8.80 \\
\hline SUA: Mean Fano factor & 0.90 & 1.29 & 1.24 & 1.01 & 1.07 & 1.10 \\
\hline SUA: Mean (Adapter - Test), spikes/sec & - & 9.23 & 14.38 & - & 6.19 & 8.37 \\
\hline MUA: Mean response, spikes/sec & 59.27 & 56.11 & 46.42 & 50.03 & 46.73 & 41.88 \\
\hline MUA: Mean standard deviation, spikes/sec & 17.75 & 17.14 & 15.14 & 16.21 & 15.28 & 14.48 \\
\hline MUA: Mean Fano factor & 1.45 & 1.44 & 1.38 & 1.45 & 1.39 & 1.40 \\
\hline MUA: Mean (Adapter - Test), spikes/sec & - & 3.16 & 12.85 & - & 3.30 & 8.15 \\
\hline
\end{tabular}

SUA, single unit recordings, $n=80$ neurons, shape distance $D=2$; MUA, multiunit recordings, $n=468$ recording sites. Test(XY) indicates test stimulus Y following adapter stimulus X.

( 1 being the preferred shape as adapter). The stippled line indicates the mean $d^{\prime}$ for the same shape pair presented as adapter. Repeated-measures ANOVA showed a significant effect of Adapter Value $(F(4,100)=4.8238, p=$ .0013). The discriminability was enhanced when the adapter was the least preferred shape (comparison adapter and test following the least preferred adapter; two-sided Wilcoxon matched pairs test: $p=.0201$ ), which is in line with Dragoi et al. (2002). However, contrary to the adaptation data in V1 (Dragoi et al., 2002), the discriminability of the test stimuli was significantly reduced when the adapter was the preferred shape (comparison adapter and test following adapter identical to the preferred shape; two-sided Wilcoxon matched pairs test: $p=.0014$ ). This agrees with the strong reduction of the $d^{\prime}$ in the Test $(\mathrm{PP}, \mathrm{PL})$ condition observed for the whole sample (Figure 2A).

To determine whether this enhanced discriminability is driven by the difference between the adapter and test stimuli or by the effectiveness of the adapter stimulus, we repeated the same analysis for the same neurons except the $d^{\prime}$ was computed for the less effective shapes again using a distance of two values. Thus, $d$ 's were computed for test stimuli that were two and four values away from the preferred shape $\mathrm{P}$ as a function of adapter value.
Repeated-measures ANOVA showed a significant effect of adapter value $(F(4,100)=4.4508, p=.0024$; Figure $3 \mathrm{~B})$. Although $d^{\prime}$ was again significantly reduced for preferred adapters (comparison adapter and test following adapter identical to the preferred shape; two-sided Wilcoxon matched pairs test: $p=.0132$ ), no enhancement was present (comparison adapter and test following the least preferred adapter; two-sided Wilcoxon matched pairs test: $p=.8093)$. These data show that both the effectiveness of the adapter and the test stimuli determine the adaptation-induced changes in $d^{\prime}$.

The effect of adapter value on the $d^{\prime}$ shown in Figure 3 are due to a combination of the effect of adaptation on response strength and response variability for the different adapter-test stimulus combinations (Table 3). The response to the most effective stimulus (value 1) presented as test increased with increasing adapter value, whereas the response to the less effective stimulus (value 5) decreased with increasing adapter value, reflecting stimulus-specific adaptation (De Baene \& Vogels, 2010; Liu et al., 2009). However, these adaptation-induced changes in response strength could not explain the enhanced discriminability for the preferred shapes when the adapters were less preferred (Figure $3 \mathrm{~A}$ ) because

Table 2. Mean Response Difference, Mean Absolute Response Difference and $d^{\prime}$ for P and L in the Different Adaptation Conditions

\begin{tabular}{|c|c|c|c|c|c|}
\hline & Adapter & $\operatorname{Test}(P \boldsymbol{P}, L \boldsymbol{L})$ & $\operatorname{Test}(P \boldsymbol{L}, L \boldsymbol{P})$ & Test $(P \boldsymbol{P}, P \boldsymbol{L})$ & $\operatorname{Test}(L \boldsymbol{L}, L \boldsymbol{P})$ \\
\hline SUA: Mean response difference $(\mathrm{P}-\mathrm{L})$, spikes/sec & 15.39 & 9.38 & 12.35 & 7.20 & 14.53 \\
\hline SUA: Mean Absolute (response difference), spikes/sec & 15.67 & 10.82 & 14.45 & 11.53 & 15.18 \\
\hline SUA: Mean $d^{\prime}$ & 1.75 & 1.35 & 1.75 & 1.38 & 1.76 \\
\hline MUA: Mean response difference $(\mathrm{P}-\mathrm{L})$, spikes/sec & 9.24 & 4.54 & 9.38 & -0.31 & 14.23 \\
\hline MUA: Mean Absolute (response difference), spikes/sec & 9.38 & 5.63 & 10.43 & 5.86 & 14.37 \\
\hline MUA: Mean $d^{\prime}$ & 0.53 & 0.38 & 0.61 & 0.38 & 0.87 \\
\hline
\end{tabular}

For the SUA, data are for a shape distance of 2 (as in Table 1). The same conventions as in Table 1 and Figure 2. 
the mean absolute response differences for these adaptation conditions were not consistently larger than those for the same stimuli presented as adapter (Table 3). This enhancement in discriminability reflected the lower standard deviations of the responses for these adaptation conditions compared with that for the adapter (Table 3).

\section{Effect of Adaptation on Object Discriminability of IT MUA}

To assess the generality of the above single unit IT data on the effect of stimulus repetition on discriminability, we examined data from an experiment in which we recorded
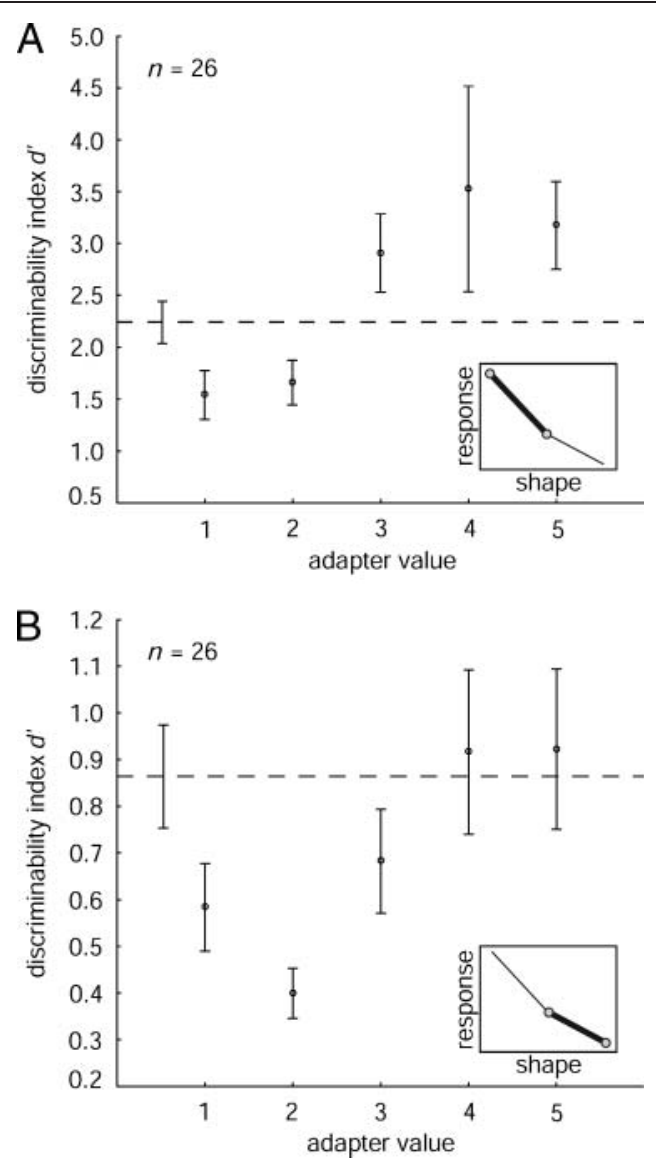

Figure 3. Mean discriminability index $d^{\prime}$ of single IT neurons plotted as a function of the adapter value of the parameterized shape. Adapter value: 1 = preferred shape; 5 = less preferred shape differing by 4 values from the adapter. (A) $d^{\prime}$ computed for the preferred shape (value $=1$ ) and the shape with value 3 (i.e., distance $=2$ ) presented as tests. (B) $d^{\prime}$ computed for the less preferred shape with value 5 and the shape with value 3 (i.e., distance $=2$ ) presented as tests. The $d^{\prime}$ value for the same shapes presented as adapters is indicated by the stippled horizontal line. Only the neurons $(n=26)$ for which the preferred shape was either an extreme (A and $\mathrm{F}$ in Figure $1 \mathrm{~A}$ ) or differed by one value from the extreme (B and $\mathrm{E}$ in Figure 1A) were considered for this analysis. Bars indicate standard errors of the mean. Insets: schematic monotonic tuning curves with stimuli used to compute $d^{\prime}$ indicated by filled, gray circles and connected by a thick black line.
MUA from up to 16 sites simultaneously with a laminar electrode located in IT during an adaptation paradigm. For each penetration, two different stimuli, A and B, were employed. Stimulus duration and ISI were $500 \mathrm{msec}$ instead of 300 msec (Figure 1B). Thirty-two electrode penetrations were made, yielding 468 responsive MUA sites (319 and 149 sites in each animal). As demonstrated by Kaliukhovich and Vogels (2012), repetition suppression was present in the first half of the response to a stimulus but nearly absent in the later half (Figure 4A). Repetition suppression was very common because $90 \%$ of the Stimulus $\times$ Responsive site combinations showed a smaller response to the test compared with the adapter stimulus in repetition trials. Since the two monkeys showed similar effects, we pooled data across the animals.

For each site, we assigned $P$ to the stimulus eliciting the largest response and $\mathrm{L}$ to the stimulus eliciting the smallest response of the two when presented as adapter. Figure $2 \mathrm{~B}$ shows the mean $d^{\prime}$ s for the MUA using the same conventions as in Figure 2A. As for the single unit data, repeated-measures ANOVA showed a significant effect of adaptation condition (main effect of adaptation condition: $\left.F(4,1868)=209.56, p=10^{-15}\right)$. Post hoc Bonferroni tests demonstrated a reduction in $d^{\prime}$ for the test stimuli in repetition trials (Test $(\mathbf{P} \mathbf{P}, \mathbf{L} \mathbf{L}))$ compared with the adapter and test stimuli in alternation trials (Test(PL, LP); each $p<.10^{-6}$ ), which agrees with the single cell data (Figure 2A). The mean $d^{\prime}$ for the test in repetition trials (Test(PP, LL)) did not differ from that obtained in the Test(PP, PL) condition. In addition, the mean $d^{\prime}$ in the Test(LL, LP) condition was significantly larger than that for the same stimuli presented as adapter or as test in alternation trials (post hoc Bonferroni test: each $p<.10^{-6}$ ). As for the single cell data, the adaptation-induced changes in $d^{\prime}$ were mainly due to changes in the response strength and not response variability (Fano Factor; Tables 1 and 2). The adaptation-induced response reduction was larger for the P compared with L stimuli presented as tests in repetition trials (Test $(\mathrm{P} \mathbf{P})$ versus Test $(\mathbf{L} \mathbf{L})$ in Table 1$)$, explaining the reduced $d^{\prime}$ in those trials compared with the adapter. The reduced $d^{\prime}$ in the Test(PP, PL) compared with the adapter condition resulted from the stronger adaptation-induced response reduction when repeating $\mathrm{P}$ than when $\mathrm{L}$ is following $\mathrm{P}$. The enhanced $d^{\prime}$ in the Test(LL, LP) condition compared with the adapter was due to the lesser response reduction when $\mathrm{P}$ followed $\mathrm{L}$ than when $\mathrm{L}$ was repeated. Thus, the opposite effects of adaptation to $\mathrm{P}$ versus $\mathrm{L}$ on $d^{\prime}$ s, reduction and enhancement, respectively, results from the stimulus-specific adaptation in IT, that is, the stronger response reduction in repetition compared with alternation trials (Table 1; De Baene \& Vogels, 2010; Liu et al., 2009). In the case of adaptation to $\mathrm{P}$, this leads to less response difference between $\mathrm{P}$ and $\mathrm{L}$ because of the large reduction of the response to the more effective stimulus $\mathrm{P}$, whereas adapting to $\mathrm{L}$ increases the response difference between $\mathrm{P}$ and $\mathrm{L}$ since the response to the less effective stimulus $\mathrm{L}$ is 
Table 3. Responses, Response Differences and Response Variability for the Stimulus Conditions and Neurons ( $n=26)$ of Figure 3

\begin{tabular}{|c|c|c|c|c|c|c|}
\hline & \multirow[b]{2}{*}{ As Adapter } & \multicolumn{5}{|c|}{$\begin{array}{c}\text { As Test following Adapter with } \\
\text { Value \# }\end{array}$} \\
\hline & & $\# 1$ & $\# 2$ & \#3 & $\# 4$ & \#5 \\
\hline Response to stimulus with value \#1, spikes/sec & 45.43 & 32.16 & 32.75 & 37.67 & 37.04 & 37.44 \\
\hline Response to stimulus with value \#3, spikes/sec & 26.43 & 18.80 & 18.05 & 18.69 & 19.19 & 18.46 \\
\hline Response to stimulus with value \#5, spikes/sec & 23.10 & 17.44 & 18.01 & 18.01 & 16.92 & 16.37 \\
\hline Mean Absolute (response difference) between stimuli \#1 and \#3, spikes/sec & 18.88 & 13.36 & 14.74 & 18.98 & 17.85 & 18.98 \\
\hline Mean standard deviation of stimuli \#1 and \#3, spikes/sec & 9.26 & 10.07 & 9.93 & 8.11 & 8.24 & 7.33 \\
\hline Mean Absolute (response difference) between stimuli \#3 and \#5, spikes/sec & 6.19 & 4.79 & 4.00 & 4.53 & 5.69 & 4.73 \\
\hline Mean standard deviation of stimuli \#3 and \#5, spikes/sec & 8.06 & 9.87 & 10.02 & 7.79 & 8.18 & 7.31 \\
\hline
\end{tabular}

Top 3 rows: Mean responses to stimuli with values 1, 3, and 5 with 1 corresponding to the preferred shape P and 3 and 5 to a shape with a distance of 2 and 4, respectively, from the preferred shape. Responses are given for the 3 stimuli presented as adapter (first column) and as test stimuli following an adapter with value \#X. Bottom 4 rows: Mean absolute difference and between-trial mean standard deviation for stimulus pairs $(1,3)$ and $(3,5)$, with mean $d^{\prime}$ depicted in Figure $3 \mathrm{~A}$ and $\mathrm{B}$, respectively.

further reduced relative to $\mathrm{P}$ (for an illustration see Figure 5). The latter enhancement will be stronger when the response to $\mathrm{P}$ is only weakly affected by $\mathrm{L}$, which was the case in the MUA experiment (Table 1) where highly dissimilar stimuli were employed. However, this crossadaptation was stronger in the single unit study (Table 1) in which the P and L stimuli were more similar, explaining why no significant enhancement was observed in the Test (LL, LP) condition in that experiment.

Images A and B can be a P and an L stimulus, respectively, for a particular neuron, whereas the reverse can be the case for another neuron. Thus, adapting to A will decrease the discriminability in neurons for which $\mathrm{A}$ is $\mathrm{P}$ while increasing the discriminability in neurons for which $\mathrm{A}$ is $\mathrm{L}$. In a population consisting of both types of neurons, these opposite effects may cancel out the overall adaptation effects on discriminability. To address this, we next determined the effects of adaptation on the object decoding accuracy of a population of neurons.

\section{Effect of Adaptation on Object Classification by IT Neuronal Populations}

Because in the second experiment we recorded in each penetration from multiple sites simultaneously, we could determine the effect of stimulus repetition on the object decoding accuracy of a small population of neurons, taking into account trial-to-trial covariations in activity. The median number of responsive MUA sites per penetration was 15.5 (range: 11-16). To determine whether classification accuracy was affected by stimulus repetition, we classified single trial spike counts of the same 250-msec duration windows as used to compute $d^{\prime}$. Figure 6A shows the mean classification accuracy, averaged across the 32 penetrations, obtained with the correlation-based classifier. The classifier was trained and tested for each of the adaptation conditions of Figure 6A separately.
Classifiers for the two adapter stimuli A and B were trained and tested in repetition and alternation trials separately. As expected, the classification accuracies for the adapter did not differ significantly between the two trial types (two-sided Wilcoxon matched pairs test: $p=.5128$ ), allowing averaging of the classification scores for the adapter stimuli in repetition and alternation trials. Likewise, classification scores for the test stimuli, A and B, when these were following an identical versus a different adapter were obtained separately for $\mathrm{AA}$ versus $\mathrm{AB}$ and $\mathrm{BB}$ versus $\mathrm{BA}$ trials but did not differ significantly (two-sided Wilcoxon matched pairs test: $p=.2865$ ) and, thus, were also averaged. There was a significant effect of adaptation condition on the classification accuracy (repeated-measures ANOVA: main effect of Stimulus Condition: $F(3,93)=$ $18.895, p=.1188 \cdot 10^{-8}$ ). The lowest classification scores ( $72 \%$ correct) were obtained for the test stimulus in repetition trials (Test(AA, BB)), which differed significantly from each of the three other conditions ( $>76 \%$ correct; post hoc Bonferroni tests: each $p<.0001$ ), in agreement with the $d^{\prime}$ data reported above. The reduction of the classification accuracy for the test stimuli in repetition compared with alternation trials was present in all but 3 of the 32 penetrations, in each animal and irrespectively of the number of responsive sites of a penetration (Figure $6 \mathrm{~B}$ ). In addition, the classification scores for the test in alternation trials (Test(AB, BA ); 79\% correct) was significantly higher than for the $\operatorname{Test}(\mathrm{AA}, \mathrm{AB})$ condition (76\% correct; post hoc Bonferroni test: $p=.0462$ ). No other differences were significant after Bonferroni correction. In general, stimulus repetition reduced the classification accuracy of the small population of neurons.

The above analyses were all based on spike counts in a relatively long analysis window (as was done in previous studies of Hansen \& Dragoi, 2011; Wang et al., 2011; Müller et al., 1999). To determine whether similar effects would be present with a finer temporal resolution, 

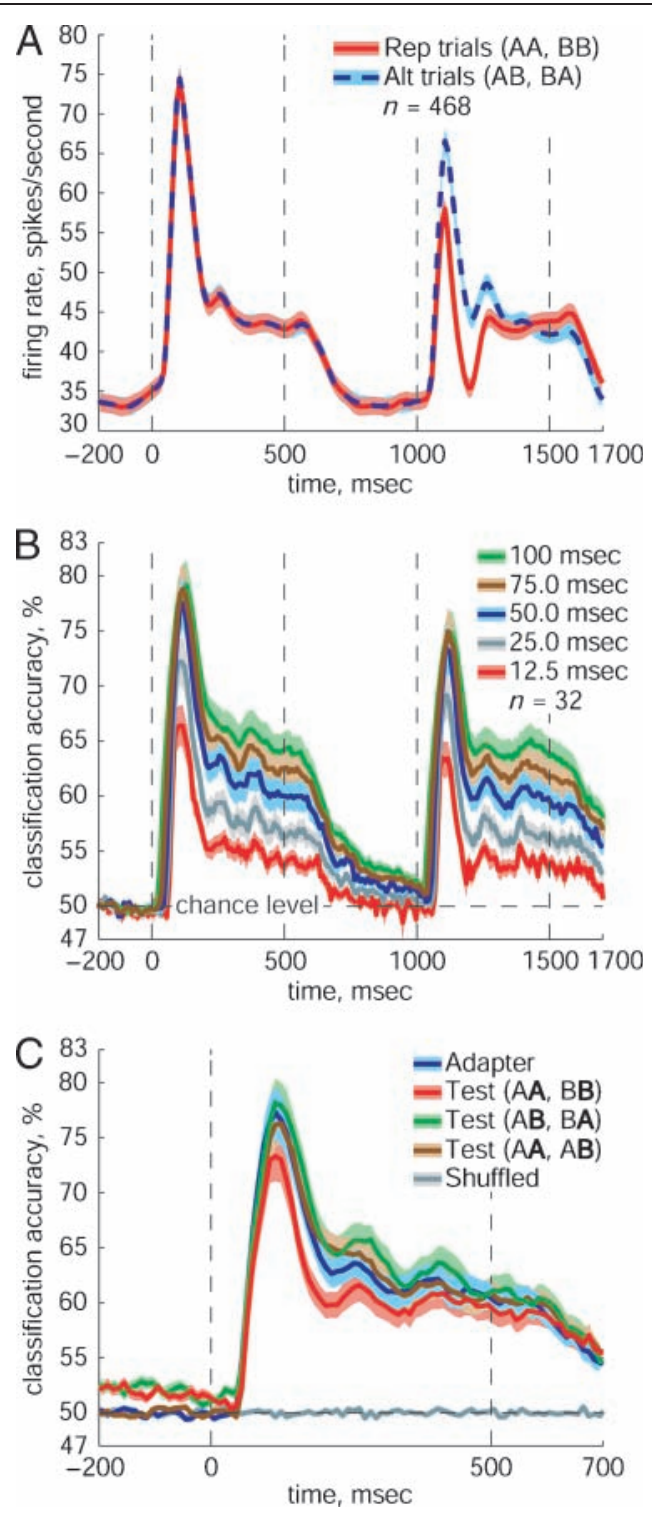

Figure 4. Time course of MUA and classification accuracy. (A) Population PSTH of the mean spiking activity, averaged across all responsive recording sites of both animals, in repetition (Rep trials) and alternation (Alt trials) trials. Responses are aligned on the onset of the adapter stimulus. Stippled vertical lines indicate stimulus on- and offsets. Firing rates were computed with a sliding window of $50 \mathrm{msec}$ with a step of $10 \mathrm{msec}$. (B) Mean classification accuracy computed with different sliding window durations (see legend) for repetition trials (AA and $\mathrm{BB}$ trials). Mean accuracies are plotted at the center of the corresponding window (e.g., accuracy for the 0-50 msec window plotted at $25 \mathrm{msec}$ ); 0 msec corresponds to the onset of the adapter stimulus. Chance level is 50\%. (C) Mean classification accuracy for four adaptation conditions (see legend), obtained with a sliding window of $50 \mathrm{msec}$ and aligned on stimulus onset. Mean classification accuracy obtained when shuffling the stimulus labels across trials of a condition (Shuffled) were computed using the same sliding windows and did not differ from the chance level (50\%). Training and testing was done for each condition and window separately using the correlation-based classifier. Vertical stippled lines indicate stimulus on- and offset. Bands indicate standard errors of the mean. we performed the classification analysis for five shorter windows with durations ranging from 12.5 to $100 \mathrm{msec}$. The time course of the mean correct classification accuracy in repetition trials is shown for the five durations of the sliding window in Figure 4B. The training and testing were performed for each window separately. Reliable classification was already present for the short window duration of 12.5 msec. The peak classification accuracy increased steeply with longer window durations up to $50 \mathrm{msec}$ at which nearly maximum performance was reached. This held for both adapter and test stimuli. Importantly, the peak classification accuracy was lower for the test compared with the adapter stimulus in the repetition trials for all examined window durations. Note that the stimulus repetition did affect the amount but not the timing of the maximum information. This contradicts the facilitation model of adaptation since that model posits faster processing of repeated stimuli (Grill-Spector et al., 2006; James \& Gauthier, 2006). Window duration had a strong effect on classification accuracy in the later, more sustained part of response for both the adapter and test stimuli. This suggests that, after the initial transient, longer integration times are needed to obtain more accurate classification, but even with an integration window of $100 \mathrm{msec}$ (and $150 \mathrm{msec}$; data not shown), the classification performance is still well below

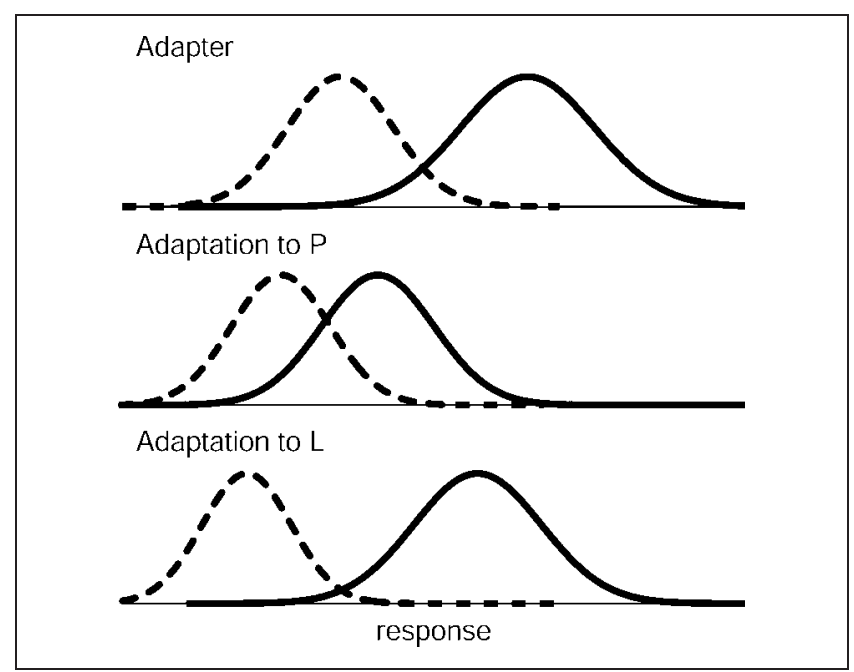

Figure 5. Illustration of effects of adaptation on neuronal discriminability. The two distributions in each row represent the responses to the $\mathrm{P}$ (solid line) and $\mathrm{L}$ (stippled line) stimuli. Top row: response distributions for stimuli presented as adapter; middle row: response distributions for stimuli when adapting to P; bottom row: response distributions for stimuli when adapting to L. Adaptation to $\mathrm{P}$ produces a larger reduction of the response to the repeated stimulus $\mathrm{P}$ than to $\mathrm{L}$, resulting in a decreased discriminability. When adapting to $\mathrm{L}$, the response to the repeated stimulus $\mathrm{L}$ is more suppressed compared with the response to $\mathrm{P}$, which enhances discriminability between them. The Fano factor was equated for all response distributions. Note that the distributions do not represent real, recorded data but serve to explain graphically the differential effects of adaptation to P and L stimuli. 

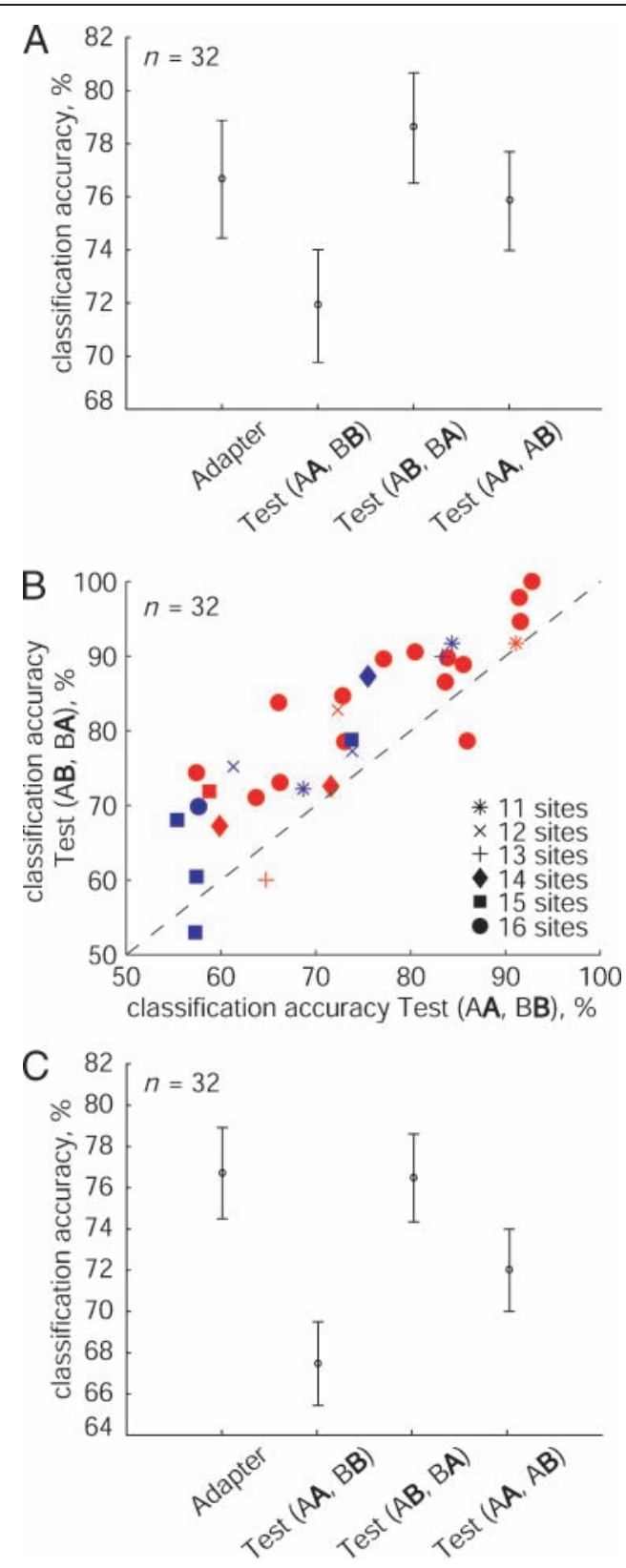

Figure 6. Classification accuracy ( $n=32$ penetrations) of MUA. (A) Mean classification plotted for four adaptation conditions: adapter stimulus, test stimulus in repetition trials (Test(AA, BB)), test stimulus in alternation trials (Test $(\mathrm{A} \mathbf{B}, \mathrm{BA})$ ), and the average of two conditions Test $(\mathrm{A} \mathbf{A}, \mathbf{A} \mathbf{B})$ and $\operatorname{Test}(\mathrm{B} \mathbf{B}, \mathrm{B} \mathbf{A})$ (here indicated by Test(AA, AB $)$ ). Classification accuracies were obtained when training and testing classifiers on the data of each condition separately. Bars indicate standard errors of the mean, and bold letters correspond to the stimuli for which classification was performed. (B) Classification accuracy for the test stimuli in alternation trials plotted against the classification accuracy for the same stimuli in repetition trials. Each point corresponds to a single penetration. Different symbols and colors label penetrations according to the number of responsive sites of a penetration and animal, respectively. The diagonal is indicated by a stippled line. (C) Mean classification plotted for four adaptation conditions when the classifiers were trained only with response vectors for the adapter stimuli and then tested for each condition. Abbreviations are the same as in A. the one obtained at the peak for a short window of 25-msec duration.

As expected from the population PSTH (Figure 4A), there was little difference in classification accuracy between adapter and test stimuli in repetition trials at the late phase of the response. This can be better appreciated in Figure $4 \mathrm{C}$, which plots the time course of the classification accuracies for the adapter and test stimuli with a sliding window of $50 \mathrm{msec}$. In addition, the time course of the test stimuli in other adaptation conditions (see above) are also shown. It is clear that the test stimulus in repetition trials produces the lowest classification, while there are few differences between the accuracies for the adapter compared with the test stimulus in the two other conditions, especially at the peak. After the peak, the classification accuracy for the test stimuli in alternation trials was larger than for the adapter (Figure 4C). A higher classification accuracy for the test stimuli in alternation trials compared with the adapter can also be seen for the longer window of 250-msec duration (Figure 6B) and was highly significant for the $d^{\prime}$ s (Figure 2B).

A noteworthy feature, but peripheral with respect to the main question of this paper, is the presence of a better than chance classification accuracy at the end of the 500-msec long ISI reflecting the weak delay activity in macaque IT when passively viewing stimuli. This can be seen clearly in Figure 4C for the plots for the test stimuli in repetition and alternation trials (it is absent for the Test(AA, AB) condition since the adapters were identical (both A or both B) in those classifications).

The correlation-based classifier is a linear classifier that performs a weighted addition of the neurons' outputs. A separate training of classifiers for the adapter and test stimuli, as was done above, thus implies that the readout of the responses of the same neurons changes between the two successive presentations in a trial. In a second set of analyses, we fixed the weights of the classifier when testing the adapter and test stimuli classifications. These adapter-based classifiers were trained using responses to the adapter stimuli only. As shown in Figure 6C, the adapter-based classifiers also produced lower classification scores for the test stimuli in repetition trials $(67 \%$ correct) compared with the same stimuli presented as an adapter (77\%; post hoc Bonferroni test: $p<10^{-6}$ ). Overall, the classification scores for the test stimuli were lower when training using only the adapter responses than when training for each condition separately. This drop also resulted in significantly lower scores in the Test(AA, AB ) condition compared with the adapter condition (post hoc Bonferroni test: $p<10^{-6}$ ).

\section{DISCUSSION}

Macaque IT neurons were worse at discriminating repeated compared with nonrepeated stimuli, at least for short-term stimulus repetitions without intervening stimuli. This reduction in discrimination capacity was 
observed in two independent experiments: for single IT units tested with parameterized shapes and IT MUA tested with complex natural objects. In addition, object classification accuracy based on the simultaneous activity of small populations of IT neurons was smaller for repeated compared with unrepeated stimuli, even for very short analysis windows. Overall, these data suggest that the representation accuracy of objects in IT is not enhanced but, rather, impaired by repetition. In some conditions in which adapter and test stimuli differed, object discriminability enhanced. The adaptation-induced changes in $d^{\prime}$ mainly covaried with differences in the adaptationinduced response reduction across the various adaptation conditions.

Weiner, Sayres, Vinberg, and Grill-Spector (2010) reported a (nonsignificant) tendency for a lower category classification accuracy of the fMRI signal for blocks of repeated compared with unrepeated stimuli in human ventral temporal cortex. It is tempting to relate this to the reduced classification accuracy for repeated versus nonrepeated images seen in both our single cell and MUA data, but the link between multivoxel pattern analysis and single cell selectivity is complicated, in particular because of the large size-compared with single unitsof the fMRI voxels. Weiner et al. (2010) observed a nonsignificant decrease in classification accuracy with repetition when using a delay of $500 \mathrm{msec}$ and no intervening stimuli, which is similar to the paradigm employed in our study. However, a significant increase in classification accuracy was present when the delays between the repeated stimuli were much longer (mean of $20 \mathrm{sec}$ ) and intervening stimuli were present. Thus, it cannot be excluded that the effects of repetition on single unit discrimination accuracy depend on ISI duration and/or the presence of intervening stimuli, which remains to be investigated in future studies. Hence, our conclusions may hold only for short-term adaptation (short ISIs without intervening stimuli).

As in previous studies of adaptation in early visual cortex (Hansen \& Dragoi, 2011; Wang et al., 2011; Gutnisky \& Dragoi, 2008; Müller et al., 1999), we examined $d^{\prime}$ of single units for stimuli that were optimized per neuron (but see Figure 3). Adaptation in IT occurs for both effective and ineffective stimuli (De Baene \& Vogels, 2010; Liu et al., 2009; McMahon \& Olson, 2007; Sawamura et al., 2006), and thus, we expect that qualitatively similar effects of adaptation on discriminability of single units are also present for ineffective stimuli (as long as the stimuli drive a neuron). The use of a laminar electrode with simultaneous recordings at several sites prevented stimulus optimization for each site, but nonetheless, the MUA data set also produced reductions of discriminability following repetition. Thus, we believe that our conclusions generalize to any arbitrary stimulus set and neuron selections.

Gotts and colleagues (Gotts, Chow, \& Martin, 2012; Gilbert, Gotts, Carver, \& Martin, 2010; Gotts, 2003) proposed that stimulus repetition enhances low-frequency synchronized neural responses that are time-locked to stimulus onset, which may improve discrimination accuracy. Kaliukhovich and Vogels (2012) found a repetitioninduced, stimulus-locked power increase around $25 \mathrm{~Hz}$ in the local field potentials (LFPs) obtained in the second experiment of the present paper (MUA recordings), but this is only in one of two monkeys. Thus, our data do not fully support the relevance of Gotts' computational scheme for the short-term repetition effect in IT cortex. Moreover, the MUA time course analysis, in particular with short time windows, captures repetition effects related to synchronized activity that is stimulus locked. Since even with a window of $12.5 \mathrm{msec}$, stimulus repetition reduced classification accuracy, it appears that a potential stimulus-locked synchronized activity cannot compensate the adaptation-induced reduction in responses. Kaliukhovich and Vogels (2012) also showed that stimulus repetition decreases MUA-LFP synchronization for spectral frequencies above $50 \mathrm{~Hz}$. Under the hypothesis that synchronization affects encoding by a coincidence detection mechanism (Fries, 2009), such a repetitioninduced decrease in synchronization should have a detrimental effect on the representation of repeated stimuli and also cannot compensate the impaired classification performance seen in our analysis.

Little is known about the effect of adaptation on stimulus discriminability in extrastriate visual areas. Wang et al. (2011) reported an increase in $d^{\prime}$ for nearby orientations after short adaptation to an orientation close to the preferred orientation of V4 neurons, which appears to differ from the decrease in $d^{\prime}$ for repeated stimuli seen here in IT. However, note that their results are difficult to compare with ours because the relationships between adapter and test orientations are unclear in the report by Wang et al. (2011). Wang et al. (2011) found a correlation between an adaptation-induced increase in gamma oscillations and the change in $d^{\prime}$. Kaliukhovich and Vogels (2012) did not find such an increase in gamma oscillations in IT in the present adaptation paradigm, which may suggest a difference between adaptation effects in V4 and IT. Krekelberg, Boynton, and van Wezel (2006) found a slight (about 2\%) improvement in speed discriminability after a 2-sec long motion adaptation in MT. However, contrary to IT (De Baene \& Vogels, 2010; Liu et al., 2009), the adaptation-induced response suppression increased in MT with increasing difference between adapter and test stimuli (Krekelberg et al., 2006). It is possible that these discrepancies among different studies, including ours, reflect differences in adaptation protocols rather than between-area differences (e.g., Wang et al., 2011 employed a short ISI of $100 \mathrm{msec}$; for further discussion, see Kaliukhovich \& Vogels, 2012).

The present data suggest that short-term adaptation in IT does not increase the classification accuracy of recently seen stimuli. This is opposite to what one might expect from repetition priming (Schacter, 1987). However, in typical priming studies, stimuli are novel (except when 
repeated), unlike this study and most other adaptation studies, in which only familiar stimuli are employed. Priming might be related to the tuning sharpening of IT and prefrontal neurons that occurs when novel stimuli become familiar (Woloszyn \& Sheinberg, 2012; Freedman, Riesenhuber, Poggio, \& Miller, 2006; Rainer \& Miller, 2000; but see McMahon \& Olson, 2007). It will be interesting to assess in future research whether the decrease in object discrimination accuracy of IT neurons following repetition is correlated with a psychophysical decrease in accuracy (see Kohn, 2007, for review of perceptual studies using simple stimuli).

What can we deduce from these data about the possible functional significance of the short-term adaptation? The discriminability of the test stimuli compared with that of the adapter stimuli was strongly reduced in repetition trials but could be enhanced in alternation trials. Because arguably IT neurons that prefer an object contribute positively to the decoding of that object (Verhoef, Vogels, \& Janssen, 2012), the enhancement in $d^{\prime}$ for more effective stimuli after adaptation to lesser effective stimuli (Figure 3A) may increase the accuracy of the object representation. Thus, given that during free viewing different parts of the same object or different objects tend to be foveated successively, short-term adaptation can enhance the coding of newly foveated objects.

In general, the marked drop in classification accuracy for repeated stimuli in IT implies that these neurons reduce their coding of recently observed stimuli compared with a stimulus that differs from a recently seen one. This might be a sensible strategy for an object identification/categorization system since an object that differs from a recently observed object needs to be identified/ categorized to produce an adaptive behavioral response but it is less required to identify/categorize de novo a recently seen object. This selective reduction of the response to repeated stimuli can be metabolically opportune. At the same time, the classification of a changed stimulus is efficient. In addition, such a stimulus-specific adaptation mechanism may result in anticipatory, predictive responses in natural action sequences such as of head rotations or other bodily actions (Perrett, Xiao, Barraclough, Keysers, \& Oram, 2009) and may contribute to short-term recognition memory (Orban \& Vogels, 1998; Miller \& Desimone, 1994).

\section{Acknowledgments}

The technical assistance of P. Kayenbergh, G. Meulemans, I. Puttemans, M. Docx, M. De Paep, W. Depuydt, and S. Verstraeten is gratefully acknowledged. We thank Dr. J. Taubert, Dr. B. Verhoef, and I. Popivanov for critical reading a draft of this paper. This work was supported by Fonds voor Wetenschappelijk Onderzoek Vlaanderen (G.0644-08), Geconcerteerde Onderzoeksactie (GOA/ 10/019), Interuniversitaire Attractiepool (IUAP), and Programma Financiering (PF 10/008). For the computation of classifiers, we used the infrastructure of the VSC-Flemish Supercomputer Center, funded by the Hercules Foundation and the Flemish Government-Department EWI.
Reprint requests should be sent to Rufin Vogels, Laboratorium voor Neuro- en Psychofysiologie, KU Leuven, Campus Gasthuisberg, O\&N 2, Herestraat 49, bus 1021, 3000 Leuven, Belgium, or via e-mail: Rufin.Vogels@med.kuleuven.be.

\section{REFERENCES}

Barlow, H. B. (1961). Possible principles underlying the transformations of sensory messages. In W. A. Rosenblith (Ed.), Sensory communication (pp. 217-234). Cambridge, MA: MIT Press.

Baylis, G. C., \& Rolls, E. T. (1987). Responses of neurons in the inferior temporal cortex in short term and serial recognition memory tasks. Experimental Brain Research, 65, 614-622.

De Baene, W., \& Vogels, R. (2010). Effects of adaptation on the stimulus selectivity of macaque inferior temporal spiking activity and local field potentials. Cerebral Cortex, 20, 2145-2165.

Desimone, R. (1996). Neural mechanisms for visual memory and their role in attention. Proceedings of the National Academy of Sciences, U.S.A., 93, 13494-13499.

Dragoi, V., Sharma, J., Miller, E. K., \& Sur, M. (2002). Dynamics of neuronal sensitivity in visual cortex and local feature discrimination. Nature Neuroscience, 5 , 883-891.

Freedman, D. J., Riesenhuber, M., Poggio, T., \& Miller, E. K. (2006). Experience-dependent sharpening of visual shape selectivity in inferior temporal cortex. Cerebral Cortex, 16, 1631-1644.

Fries, P. (2009). Neuronal gamma-band synchronization as a fundamental process in cortical computation. Annual Review of Neuroscience, 32, 209-224.

Gilbert, J. R., Gotts, S. J., Carver, F. W., \& Martin, A. (2010) Object repetition leads to local increases in the temporal coordination of neural responses. Frontiers in Human Neuroscience, 4, 30.

Gotts, S. J. (2003). Mechanisms underlying enbanced processing efficiency in neural systems (Ph.D. thesis). Pittsburgh, PA: Carnegie Mellon University.

Gotts, S. J., Chow, C. C., \& Martin, A. (2012). Repetition priming and repetition suppression: Multiple mechanisms in need of testing. Cognitive Neuroscience, 3, 227-259.

Grill-Spector, K., Henson, R., \& Martin, A. (2006). Repetition and the brain: Neural models of stimulus-specific effects. Trends in Cognitive Sciences, 10, 14-23.

Gross, C. G., Bender, D. B., \& Rocha-Miranda, C. E. (1969). Visual receptive fields of neurons in inferotemporal cortex of the monkey. Science, 166, 1303-1306.

Gross, C. G., Schiller, P. H., Wells, C., \& Gerstein, G. L. (1967). Single-unit activity in temporal association cortex of the monkey. Journal of Neurophysiology, 30, 833-843.

Gutnisky, D. A., \& Dragoi, V. (2008). Adaptive coding of visual information in neural populations. Nature, 452 , 220-224.

Hansen, B. J., \& Dragoi, V. (2011). Adaptation-induced synchronization in laminar cortical circuits. Proceedings of the National Academy of Sciences, U.S.A., 108, 10720-10725.

James, T. W., \& Gauthier, I. (2006). Repetition-induced changes in BOLD response reflect accumulation of neural activity. Human Brain Mapping, 27, 37-46.

Kaliukhovich, D. A., \& Vogels, R. (2011). Stimulus repetition probability does not affect repetition suppression in macaque inferior temporal cortex. Cerebral Cortex, 21, $1547-1558$ 
Kaliukhovich, D. A., \& Vogels, R. (2012). Stimulus repetition affects both strength and synchrony of macaque inferior temporal cortical activity. Journal of Neurophysiology, 107, 3509-3527.

Kohn, A. (2007). Visual adaptation: Physiology, mechanisms, and functional benefits. Journal of Neurophysiology, 97, 3155-3164.

Krekelberg, B., Boynton, G. M., \& van Wezel, R. J. (2006). Adaptation: From single cells to BOLD signals. Trends in Neurosciences, 29, 250-256.

Liu, Y., Murray, S. O., \& Jagadeesh, B. (2009). Time course and stimulus dependence of repetition-induced response suppression in inferotemporal cortex. Journal of Neurophysiology, 101, 418-436.

McMahon, D. B., \& Olson, C. R. (2007). Repetition suppression in monkey inferotemporal cortex: Relation to behavioral priming. Journal of Neurophysiology, 97, 3532-3543.

Meyers, E. M., Freedman, D. J., Kreiman, G., Miller, E. K., \& Poggio, T. (2008). Dynamic population coding of category information in inferior temporal and prefrontal cortex. Journal of Neurophysiology, 100, 1407-1419.

Miller, E. K., \& Desimone, R. (1994). Parallel neuronal mechanisms for short-term memory. Science, 263, $520-522$.

Miller, E. K., Gochin, P. M., \& Gross, C. G. (1991). Habituation-like decrease in the responses of neurons in inferior temporal cortex of the macaque. Visual Neuroscience, 7, 357-362.

Miller, E. K., Li, L., \& Desimone, R. (1991). A neural mechanism for working and recognition memory in inferior temporal cortex. Science, 254, 1377-1379.

Müller, J. R., Metha, A. B., Krauskopf, J., \& Lennie, P. (1999). Rapid adaptation in visual cortex to the structure of images. Science, 285, 1405-1408.
Orban, G. A., \& Vogels, R. (1998). The neuronal machinery involved in successive orientation discrimination. Progress in Neurobiology, 55, 117-147.

Perrett, D. I., Xiao, D., Barraclough, N. E., Keysers, C., \& Oram, M. W. (2009). Seeing the future: Natural image sequences produce "anticipatory" neuronal activity and bias perceptual report. Quarterly Journal of Experimental Psychology: (2006), 62, 2081-2104.

Rainer, G., \& Miller, E. K. (2000). Effects of visual experience on the representation of objects in the prefrontal cortex. Neuron, 27, 179-189.

Ringo, J. L. (1996). Stimulus specific adaptation in inferior temporal and medial temporal cortex of the monkey. Behavioural Brain Research, 76, 191-197.

Sawamura, H., Orban, G. A., \& Vogels, R. (2006). Selectivity of neuronal adaptation does not match response selectivity: A single-cell study of the fMRI adaptation paradigm. Neuron, 49, 307-318.

Schacter, D. L. (1987). Implicit memory: History and current status. Journal of Experimental Psychology: Learning Memory and Cognition, 13, 501-518.

Verhoef, B. E., Vogels, R., \& Janssen, P. (2012). Inferotemporal cortex subserves three-dimensional structure categorization. Neuron, 73, 171-182.

Wang, Y., Iliescu, B. F., Ma, J., Josic, K., \& Dragoi, V. (2011). Adaptive changes in neuronal synchronization in macaque V4. Journal of Neuroscience, 31, 13204-13213.

Weiner, K. S., Sayres, R., Vinberg, J., \& Grill-Spector, K. (2010). fMRI-adaptation and category selectivity in human ventral temporal cortex: Regional differences across time scales. Journal of Neurophysiology, 103, 3349-3365.

Woloszyn, L., \& Sheinberg, D. L. (2012). Effects of long-term visual experience on responses of distinct classes of single units in inferior temporal cortex. Neuron, 74, 193-205. 